\title{
Cycle decompositions of complete digraphs
}

\author{
A. C. Burgess * \\ Department of Mathematics and Statistics \\ University of New Brunswick \\ Saint John, NB E2L 4L5 \\ Canada
}

\author{
P. Danziger ${ }^{\dagger}$ \\ Department of Mathematics \\ Ryerson University \\ Toronto, ON M5B 2K3 \\ Canada
}

\author{
M. T. Javed \\ Department of Computer Science \\ Ryerson University \\ Toronto, ON M5B 2K3 \\ Canada
}

Submitted: Oct 3, 2018; Accepted: Oct 21, 2020; Published: Feb 12, 2021

(C) The authors. Released under the CC BY-ND license (International 4.0).

\begin{abstract}
In this paper, we consider the problem of decomposing the complete directed graph $K_{n}^{*}$ into cycles of given lengths. We consider general necessary conditions for a directed cycle decomposition of $K_{n}^{*}$ into $t$ cycles of lengths $m_{1}, m_{2}, \ldots, m_{t}$ to exist and provide a powerful construction for creating such decompositions in the case where there is one 'large' cycle. Finally, we give a complete solution in the case when there are exactly three cycles of lengths $\alpha, \beta, \gamma \neq 2$. Somewhat surprisingly, the general necessary conditions turn out not to be sufficient in this case. In particular, when $\gamma=n, \alpha+\beta>n+2$ and $\alpha+\beta \equiv n(\bmod 4), K_{n}^{*}$ is not decomposable.
\end{abstract}

Mathematics Subject Classifications: 05C51, 05B30, 05C20, 05C38

\section{Introduction}

Let $G$ be a graph and $\mathcal{H}=\left\{H_{1}, H_{2}, \ldots, H_{r}\right\}$ be a collection of subgraphs of $G$. We say that $\mathcal{H}$ decomposes $G$ if the edges of the graphs in $\mathcal{H}$ partition the edges of $G$. In this case, we write $G=H_{1} \oplus H_{2} \oplus \cdots \oplus H_{r}$. If $H_{1} \cong H_{2} \cong \ldots \cong H_{r} \cong H$, we refer to an

\footnotetext{
*Supported by NSERC Discovery Grant RGPIN-2019-04328.

${ }^{\dagger}$ Supported by NSERC Discovery Grant RGPIN-2016-04178.
} 
$H$-decomposition of $G$; in this case, we call the decomposition uniform. An oft-studied case is that $G$ is the complete graph $K_{n}$ and each element of $\mathcal{H}$ is a cycle. It is easy to see that if $n$ is even, then there can be no cycle decomposition of $K_{n}$. In this case, it is common instead to consider $K_{n}-I$ the complete graph with the edges of a 1 -factor $I$ removed. We will use $C_{m}$ to denote a cycle of length $m$.

The existence question for cycle decompositions of complete graphs has a history dating to the mid-1800s; among the first landmark results were Kirkman's 1847 proof that $K_{n}$ has a $C_{3}$-decomposition if and only if $n \equiv 1$ or $3(\bmod 6)$ [6] and Walecki's construction of Hamilton cycle decompositions of the complete graph [8]. The existence problem for uniform cycle decomposition of $K_{n}$ and $K_{n}-I$ was only settled over a century later $[2,9]$.

Theorem $1([2,9])$. Let $n, k \geqslant 3$ be integers. There exists a $C_{m}$-decomposition of $K_{n}$ if and only if $n$ is odd and $k \mid \frac{n(n-1)}{2}$. There exists a $C_{m}$-decomposition of $K_{n}-I$ if and only if $n$ is even and $m \mid \frac{n(n-2)}{2}$.

A more general question is the existence of possibly non-uniform cycle decompositions of $K_{n}$ or $K_{n}-I$. It was conjectured by Alspach [1] in 1981 that the obvious necessary conditions for the existence of such a decomposition were sufficient. Alspach's conjecture was finally verified in a 2014 paper by Bryant, Horsley and Pettersson [5].

Theorem 2 ([5]). Let $n \geqslant 3$ be an integer, and let $G_{n}$ denote $K_{n}$ if $n$ is odd, and $K_{n}-I$ if $n$ is even. Let $\mathcal{H}=\left\{C_{m_{1}}, C_{m_{2}}, \ldots, C_{m_{r}}\right\}$. Then $\mathcal{H}$ decomposes $G_{n}$ if and only if $3 \leqslant m_{i} \leqslant n$ for each $i=1,2, \ldots, r$ and $m_{1}+m_{2}+\cdots+m_{r}=n\left\lfloor\frac{n-1}{2}\right\rfloor$.

More recently, Bryant, Horsley, Maenhaut and Smith [4] have extended this result, finding necessary and sufficient conditions for the existence of a cycle decomposition of the complete multigraph $\lambda K_{n}$.

Theorem 3 ([4]). Let $\mathcal{C}=\left\{C_{m_{1}}, C_{m_{2}}, \ldots, C_{m_{r}}\right\}$. Then $\mathcal{C}$ decomposes $\lambda K_{n}$ if and only if the following conditions all hold:

1. $\lambda(n-1)$ is even;

2. $2 \leqslant m_{1}, m_{2}, \ldots, m_{r} \leqslant n$;

3. $m_{1}+m_{2}+\cdots+m_{r}=\lambda\left(\begin{array}{l}n \\ 2\end{array}\right)$;

4. $\max \left(m_{1}, m_{2}, \ldots, m_{r}\right)+r-2 \leqslant \frac{\lambda}{2}\left(\begin{array}{l}n \\ 2\end{array}\right)$ when $\lambda$ is even; and

5. $\sum_{m_{i}=2} m_{i} \leqslant(\lambda-1)\left(\begin{array}{l}n \\ 2\end{array}\right)$ when $\lambda$ is odd.

Also, $\mathcal{C}$ decomposes $\lambda K_{n}-I$ if and only if the following conditions all hold:

1. $\lambda(n-1)$ is odd;

2. $2 \leqslant m_{1}, m_{2}, \ldots, m_{r} \leqslant n$; 
3. $m_{1}+m_{2}+\cdots+m_{r}=\lambda\left(\begin{array}{l}n \\ 2\end{array}\right)-\frac{n}{2}$; and

4. $\sum_{m_{i}=2} m_{i} \leqslant(\lambda-1)\left(\begin{array}{l}n \\ 2\end{array}\right)$.

In this paper, we consider an alternative generalization of Alspach's conjecture, to cycle decompositions of the complete symmetric digraph.

For a graph $G$, we let $G^{*}$ denote the digraph formed from $G$ by replacing each edge $\{x, y\}$ with two arcs $x y$ and $y x$. In particular, $K_{n}^{*}$ denotes the complete symmetric digraph on $n$ vertices. We will use $\vec{P}_{k}$ to denote a directed path on $k$ vertices, and $\vec{C}_{k}$ a directed cycle of length $k$. We use $\left[u_{1}, u_{2}, \ldots, u_{k}\right]$ to denote a directed path with $\operatorname{arcs} u_{1} u_{2}, u_{2} u_{3}, \ldots, u_{k-1} u_{k}$ and $\left(u_{1}, u_{2}, \ldots, u_{k}\right)$ to denote a directed cycle with arcs $u_{1} u_{2}, u_{2} u_{3}, \ldots, u_{k-1} u_{k}$ together with $u_{k} u_{1}$.

The case of uniform cycle decomposition of $K_{n}^{*}$ was settled by Alspach, Gavlas, Šajna and Verrall [3].

Theorem 4 ([3]). Let $n, m \geqslant 2$ be integers. There is a $\vec{C}_{m}$-decomposition of $K_{n}^{*}$ if and only if $m \mid n(n-1)$ and $(n, m) \notin\{(4,4),(6,3),(6,6)\}$.

Let $\mathcal{C}$ denote a collection of $r$ directed cycles of lengths $m_{1}, m_{2}, \ldots, m_{r}$. It is clear that by ignoring the direction on the arcs, any $\mathcal{C}$-decomposition of $K_{n}^{*}$ yields a $\mathcal{C}$-decomposition of $2 K_{n}$. Thus, any such decomposition must satisfy the conditions of Theorem 3 with $\lambda=2$, giving the following necessary conditions:

Lemma 5. Let $\mathcal{C}=\left\{\vec{C}_{m_{1}}, \vec{C}_{m_{2}}, \ldots, \vec{C}_{m_{r}}\right\}$. If $\mathcal{C}$ decomposes $K_{n}^{*}$, then:

1. $2 \leqslant m_{1}, m_{2}, \ldots, m_{r} \leqslant n ;$

2. $m_{1}+m_{2}+\cdots+m_{r}=n(n-1)$; and

3. $\max \left(m_{1}, m_{2}, \ldots, m_{r}\right)+r-2 \leqslant\left(\begin{array}{l}n \\ 2\end{array}\right)$

In light of Theorem 3, one possible method of constructing directed cycle decompositions of $K_{n}^{*}$ would be to orient the edges in a cycle decomposition of $2 K_{n}$. However, it is worth noting that the methods of [4] for $2 K_{n}$ do not in general produce decompositions whose cycles can be oriented to give a directed cycle decomposition of $K_{n}^{*}$. In fact, as we shall see in Theorem 24, the necessary conditions stated in Lemma 5 are not also sufficient in all cases.

Definition 6. A nondecreasing list $M=\left(m_{1}, m_{2}, \ldots, m_{r}\right)$ of nonnegative integers will be called $n$-admissible if it satisfies conditions 1-3 of Lemma 5. We refer to a directed cycle decomposition of $K_{n}^{*}$ corresponding to the admissible list $M$ as an $M$-decomposition of $K_{n}^{*}$

Using the notation of [4] and [5], for a given $n$-admissible list $M$, we let $\nu_{i}(M)$ denote the number of instances of $i$ in $M$. It is worth noting that one of the major differences in decomposing $K_{n}^{*}$ (or $\lambda K_{n}$ ) rather than $K_{n}$ is the possibility of cycles of length 2 . Further, if there are cycles of length 2 in a decomposition of $K_{n}^{*}$, removing them corresponds to 
removing edges from the underlying undirected graph, $K_{n}$. Thus directed cycle decomposition of $K_{n}^{*}$ can be seen as a directed cycle decomposition of $G^{*}$ into cycles of lengths greater than 2 , where $G$ is an arbitrary graph of order $n$. This observation leads to a strategy for decomposing $K_{n}^{*}$.

Definition 7. We say that a directed cycle $\vec{C}$ decouples the arc $x y$ if $\vec{C}$ contains $y x$ but not $x y$. If $\mathcal{C}=\left\{\vec{C}_{m_{1}}, \vec{C}_{m_{2}}, \ldots, \vec{C}_{m_{r}}\right\}$ is a collection of pairwise edge-disjoint cycles, we say that an arc $x y$ is decoupled by $\mathcal{C}$ if $y x$ is contained in a cycle of $\mathcal{C}$ but $x y$ is not.

The following is an obvious consequence of Definition 7 .

Lemma 8. Let $\mathcal{C}=\left\{\vec{C}_{m_{1}}, \vec{C}_{m_{2}}, \ldots, \vec{C}_{m_{r}}\right\}$ be a set of pairwise edge-disjoint directed cycles in $K_{n}^{*}$. There is a $\vec{C}_{2}$-decomposition of $K_{n}^{*}-\mathcal{C}$ if and only if $\mathcal{C}$ leaves no arc decoupled.

For convenience of notation, we will henceforth let $\nu=\nu_{2}(M)$ denote the number of cycles of length 2 in the list $M$. In light of Lemma 8, it will sometimes be convenient to list only the cycle lengths which are greater than 2 , particularly when a decomposition is to contain many 2-cycles. Therefore, we make the following definition.

Definition 9. Let $M$ be an $n$-admissible list. The associated canonical list is the nondecreasing list $\hat{M}$ formed from $M$ by removing all instances of 2 from $M$. Note that the sum of the entries in $\hat{M}$ is $n(n-1)-2 \nu$.

In this paper, we give further results on cycle decompositions of $K_{n}^{*}$. In Section 2, we give general constructions and show sufficiency of the necessary conditions in certain cases. In Section 3, we determine necessary and sufficient conditions for the existence of an $M$-decomposition of $K_{n}^{*}$ when $\nu \geqslant|M|-3$, summarized in Theorem 24. As we will see, the necessary conditions of Lemma 5 turn out not to be sufficient in general. In particular, in Lemma 23 we exhibit a family of $n$-admissible lists for which $K_{n}^{*}$ admits no $M$-decomposition.

\section{General constructions}

We begin by noting some easy consequences of conditions 2 and 3 of Lemma 5 .

Lemma 10. Let $M$ be an n-admissible list with associated canonical list $\hat{M}=\left(m_{1}\right.$, $\left.\ldots, m_{r}\right)$. Then

1. $m_{1}+\cdots+m_{r-1} \equiv m_{r}(\bmod 2)$

2. $m_{1}+\cdots+m_{r-1} \geqslant m_{r}+2(r-2)$. Hence when $r \geqslant 3, m_{1}+\cdots+m_{r-1}>m_{r}$.

Conversely, given a list $\hat{M}=\left(m_{1}, \ldots, m_{r}\right)$ with $3 \leqslant m_{i} \leqslant n$ for each $i \in\{1, \ldots, r\}$ and $m_{1}+\cdots+m_{r} \leqslant n(n-1)$, if $\hat{M}$ satisfies conditions 1 and 2 above, then $\hat{M}$ is the associated canonical list of some $n$-admissible list $M$. 
Note that the case where equality holds in condition 2 corresponds with the case where equality holds in condition 3 of Lemma 5.

We now give some constructions of cycle decompositions of $K_{n}^{*}$ from known decompositions of complete graphs or complete symmetric digraphs.

Theorem 11. Let $M=\left(m_{1}, m_{2}, \ldots, m_{r}\right)$ be an $n$-admissible list, where $\nu_{k}(M)$ is even for each $3 \leqslant k \leqslant n$. If either

1. $n$ is odd and either $\nu=0$ or $\nu \geqslant 3$; or

2. $n$ is even and either $\nu=\frac{n}{2}$ or $\nu \geqslant \frac{n}{2}+3$,

then $K_{n}^{*}$ is $M$-decomposable.

Proof. We form a new list $M^{\prime}$ which decomposes $K_{n}$. We then form the corresponding directed graph $K_{n}^{*}$, using some of the cycles from $K_{n}$ to form 2-cycles in $K_{n}^{*}$. We consider two cases according to the parity of $n$.

First, suppose that $n$ is odd. Let $s \in\{0,4,5\}$ be such that $\nu \equiv s(\bmod 3)$. Let $M^{\prime}$ be the nondecreasing list satisfying that, for $i \in\{3,4, \ldots, n\}$,

$$
\nu_{i}\left(M^{\prime}\right)= \begin{cases}\frac{1}{2} \nu_{3}(M)+\frac{1}{3}(\nu-s), & \text { if } i=3 \\ \frac{1}{2} \nu_{s}(M)+1, & \text { if } i=s \neq 0 \\ \frac{1}{2} \nu_{i}(M), & \text { otherwise }\end{cases}
$$

Since $m_{1}+m_{2}+\cdots m_{r}=n(n-1)$, it follows that the sum of the entries of $M^{\prime}$ is $\left(\begin{array}{l}n \\ 2\end{array}\right)$, and so by Theorem $2, K_{n}$ is $M^{\prime}$-decomposable. From such a decomposition, take $\frac{\nu_{i}(M)}{2}$ cycles of length $i$ for each $3 \leqslant i \leqslant n$, and orient them both ways to obtain $\nu_{i}(M)$ directed cycles of length $i$. Each edge in the remaining cycles can be directed both ways to form a directed 2-cycle, giving $\nu_{2}(M)$ in total. It is easy to see that we obtain an $M$-decomposition of $K_{n}^{*}$.

The case where $n$ is even is similar. Again, let $s \in\{0,4,5\}$ such that $\nu \equiv s+\frac{n}{2}$ $(\bmod 3)$. Let $M^{\prime}$ be the nondecreasing list satisfying that, for $i \in\{3,4, \ldots, n\}$

$$
\nu_{i}\left(M^{\prime}\right)= \begin{cases}\frac{1}{2} \nu_{3}(M)+\frac{1}{3}\left(\nu-s-\frac{n}{2}\right), & \text { if } i=3 \\ \frac{1}{2} \nu_{s}(M)+1, & \text { if } i=s \neq 0 \\ \frac{1}{2} \nu_{i}(M), & \text { otherwise. }\end{cases}
$$

In this case, we note that $K_{n}-I$ is $M^{\prime}$-decomposable, and proceed as before, except that the edges of the 1-factor $I$ are also directed both ways to form $\frac{n}{2}$ directed 2-cycles.

Lemma 12. Let $M$ be an $n$-admissible list and $M^{\prime}$ an $n^{\prime}$-admissible list, where $n<n^{\prime}$. If $\nu_{k}(M)=\nu_{k}\left(M^{\prime}\right)$ for each $k \geqslant 3$ and there exists an $M$-decomposition of $K_{n}^{*}$, then there exists an $M^{\prime}$-decomposition of $K_{n^{\prime}}^{*}$. 
Proof. Decompose $K_{n^{\prime}}^{*}=K_{n}^{*} \oplus K_{n^{\prime}-n}^{*} \oplus K_{n, n^{\prime}-n}^{*}$. Decomposing $K_{n}^{*}$ into directed cycles of lengths given in $M$, it is easy to see that no arc of $K_{n^{\prime}}^{*}$ is left decoupled. Since all remaining directed cycles to be formed are of length 2 , the result follows by Lemma 8 .

In the remainder of this section, we will give constructions which give directed cycle decomposition in a wide array of cases. Before proceeding, we note the following result which settles existence in the case that the canonical list has size at most 2.

Theorem 13. Let $M=\left(m_{1}, m_{2}, \ldots, m_{r}\right)$. Suppose $M$ has at most two cycle lengths other than 2, i.e. $\nu \geqslant r-2$. There is an $M$-decomposition of $K_{n}^{*}$ if and only if $M$ is $n$-admissible.

Proof. It is easy to see that if $\nu=r$ (i.e. $M=(2,2, \ldots, 2)$ ), an $M$-decomposition of $K_{n}^{*}$ exists. Also, note that conditions 2 and 3 of Lemma 5 imply that no $n$-admissible list has $\nu=r-1$.

Finally, if $M$ is an $n$-admissible list with $\nu=r-2$, its canonical list has the form $\hat{M}=\left(m_{r-1}, m_{r}\right)$. By condition 3 of Lemma 5 and since $M$ is non-decreasing, $m_{r-1}+r-2 \leqslant$ $m_{r}+r-2 \leqslant \frac{n(n-1)}{2}$. But condition 2 implies that $2(r-2)+m_{r-1}+m_{r}=n(n-1)$, i.e. $\left(m_{r-1}+r-2\right)+\left(m_{r}+r-2\right)=n(n-1)$, so it must be that $m_{r-1}+r-2=m_{r}+r-2=\frac{n(n-1)}{2}$, and hence $m_{r-1}=m_{r}$. In this case, it is easy to see that an $M$-decomposition of $K_{n}^{*}$ exists.

Recall that condition 3 of Lemma 5 states that in an $n$-admissible list $M$ of size $t$ with maximum entry $m$, we have that $m+t-2 \leqslant\left(\begin{array}{l}n \\ 2\end{array}\right)$. Written in terms of the canonical list $\hat{M}=\left(m_{1}, m_{2}, \ldots, m_{r}\right)$, the total number of cycles is $t=r+\nu$, so this condition becomes $m_{r}+r+\nu-2 \leqslant\left(\begin{array}{l}n \\ 2\end{array}\right)$. The next result shows that if equality holds, then an $M$-decomposition does indeed exist.

Lemma 14. Let $M$ be an $n$-admissible list with associated canonical list $\hat{M}=\left(m_{1}, m_{2}\right.$, $\left.\ldots, m_{r}\right)$. If

$$
m_{r}+r+\nu-2=\left(\begin{array}{l}
n \\
2
\end{array}\right)
$$

then $K_{n}^{*}$ is $M$-decomposable.

Proof. First, suppose that $m_{r-1}=m_{r}$. Since $M$ is $n$-admissible and contains $\nu$ entries equal to 2 , counting edges we have that

$2 \nu+m_{1}+m_{2}+\cdots+m_{r}=2 \nu+m_{1}+m_{2}+\cdots+m_{r-2}+2 m_{r}=n(n-1)=2\left(m_{r}+r+\nu-2\right)$.

Rearranging gives $m_{1}+m_{2}+\cdots+m_{r-2}=2(r-2)$, and since $m_{i} \geqslant 3$ for each $i \in$ $\{1,2, \ldots, r-2\}$, it follows that $r=2$, which was dealt with in Theorem 13 .

Now suppose $m_{r-1}<m_{r}$. Let the vertex set of $K_{n}^{*}$ be $\mathbb{Z}_{n}$. We form the cycles of lengths $m_{1}, m_{2}, \ldots, m_{r}$. An illustration of this construction can be found in Figure 1. 


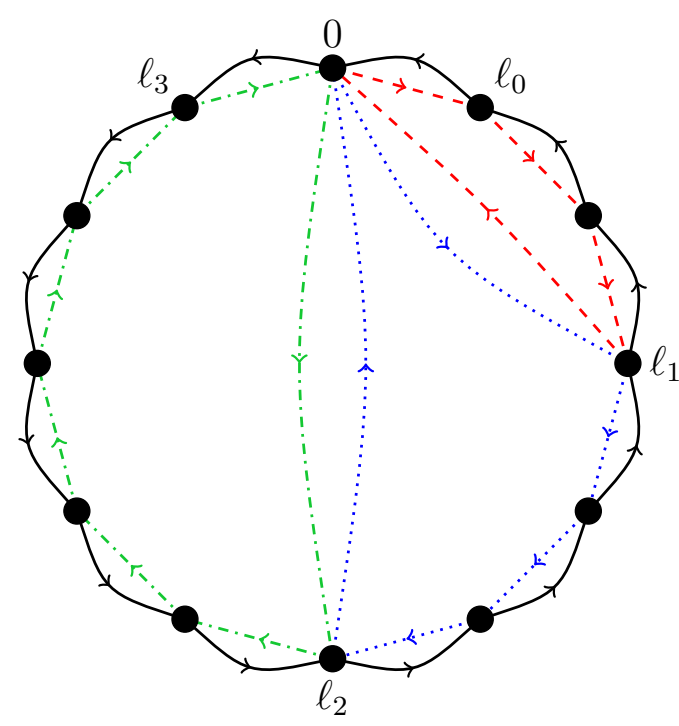

Figure 1: A $(2, \ldots, 2,4,5,7,12)$-decomposition of $K_{12}^{*}$

Let $\vec{C}_{m_{r}}=\left(m_{r}-1, m_{r}-2, \ldots, 2,1,0\right)$ be the directed cycle of length $m_{r}$. Define $\ell_{0}=1, \ell_{r-1}=m_{r}-1$, and for $i=1,2, \ldots, r-2$, let $\ell_{i}=\left(\sum_{j=1}^{i} m_{j}\right)-(2 i-1)$. Note that, since $n(n-1)=2\left(m_{r}+r+\nu-2\right)=2 \nu+m_{1}+m_{2}+\cdots+m_{r}$,

$$
\begin{aligned}
\ell_{r-2} & =\left(m_{1}+m_{2}+\cdots+m_{r-2}\right)-(2(r-2)-1) \\
& =\left(m_{r}-m_{r-1}+2 r-4\right)-(2 r-5) \\
& =m_{r}-m_{r-1}+1 .
\end{aligned}
$$

Hence, $\ell_{i}-\ell_{i-1}=m_{i}-2$ for each $i=1, \ldots, r-1$. For $i=1,2, \ldots, r-1$, define the $m_{i}$-cycle

$$
\vec{C}_{m_{i}}=\left(0, \ell_{i-1}, \ell_{i-1}+1, \ell_{i-1}+2, \ldots, \ell_{i}\right) .
$$

It is easy to verify that $\vec{C}_{m_{i}}$ has length $\ell_{i}-\ell_{i-1}+2=m_{i}$.

Note that the cycles $\vec{C}_{m_{1}}, \ldots, \vec{C}_{m_{r-1}}, \vec{C}_{m_{r}}$ leave no decoupled arc, and the result follows by Lemma 8 .

Theorem 15. Let $M$ be an n-admissible list with associated canonical list $\hat{M}=$ $\left(m_{1}, m_{2}, \ldots, m_{r}\right)$. Let $S=\left(s_{0}, s_{1}, \ldots, s_{r-2}, s_{r-1}, s_{r}\right)$ be a sequence of non-negative integers with $s_{0}=s_{r-1}=s_{r}=0$, and let $s$ be the sum of the entries of $S$. If $M$ is an $n$-admissible list such that:

1. $m_{r}+r+\nu-2=\left(\begin{array}{l}n \\ 2\end{array}\right)$; and

2. $m_{r}+s \leqslant n$,

then $K_{n}^{*}$ is $M^{\prime}$-decomposable, where $\hat{M}^{\prime}=\left(m_{\sigma(1)}^{\prime}, m_{\sigma(2)}^{\prime}, \ldots, m_{\sigma(r)}^{\prime}\right)$ such that $m_{i}^{\prime}=m_{i}+$ $s_{i-1}+s_{i}$ for each $i \in\{1, \ldots, r\}$ and $\sigma$ is a permutation which ensures that $\hat{M}^{\prime}$ is in nondecreasing order. 
Proof. We proceed in a similar manner to the proof of Lemma 14, except that we add $s$ vertices $x_{1}, \ldots, x_{s}$ to the cycles in $\hat{M}$, and modify the cycles $\vec{C}_{m_{i}}, 1 \leqslant i \leqslant r$, from that lemma as follows.

Let $\ell_{i}$ and $\vec{C}_{m_{r}}$ be as defined as in the proof of Lemma 14. For $i \in\{0,1, \ldots, r\}$, let $s_{i}^{\prime}=s_{0}+s_{1}+\cdots+s_{i}$. We define directed paths

$$
P_{i}=\left[0, x_{s_{i-1}^{\prime}+1}, x_{s_{i-1}^{\prime}+2}, \ldots, x_{s_{i}^{\prime}}, \ell_{i}\right] .
$$

Note that if $s_{i}=0$, we take $P_{i}=\left[0, \ell_{i}\right]$ of length 1 . We also define $Q_{i}$ to be the reversal of $P_{i}$, i.e. if $s_{i} \neq 0$ then

$$
Q_{i}=\left[\ell_{i}, x_{s_{i}^{\prime}}, x_{s_{i}^{\prime}-1}, \ldots, x_{s_{i-1}^{\prime}+1}, 0\right],
$$

and $Q_{i}=\left[\ell_{i}, 0\right]$ otherwise. Now, define $\vec{C}_{m_{1}}$ as the concatenation of the path $\left[0,1, \ldots, \ell_{1}\right]$ and $Q_{1}$. For $i \in\{2, \ldots, r-2\}$, define $\vec{C}_{m_{i}}$ as the concatenation of the paths $P_{i-1}$, $\left[\ell_{i-1}, \ell_{i-1}+1, \ldots, \ell_{i}\right]$ and $Q_{i}$. Finally, define $\vec{C}_{m_{r-1}}$ as the concatenation of $P_{r-2}$ and $\left[\ell_{r-2}, \ell_{r-2}+1, \ldots, \ell_{r-1}, 0\right]$ (recall that $\ell_{r-1}=m_{r}-1$ ). See Figure 2.

It is clear that these cycles leave no decoupled arcs, so the result follows by Lemma 8.

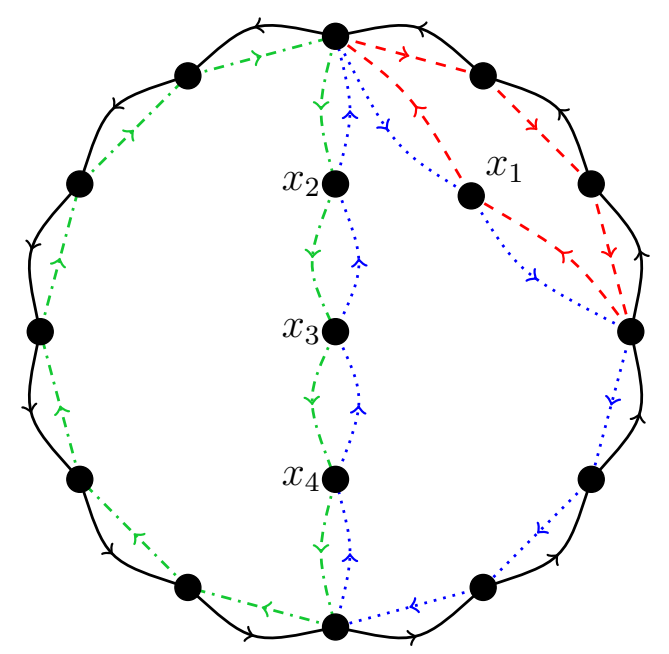

Figure 2: A $(2, \ldots, 2,5,9,10,12)$-decomposition of $K_{16}^{*}$ formed using Theorem 15 with $\hat{M}=(4,5,7,12), S=(0,1,3,0,0)$.

Note that although the statements of Lemma 14 and Theorem 15 are written so that the cycle lengths $m_{1}, \ldots, m_{r}$ are in non-decreasing order, this is not actually required in the proof, as long as $m_{r} \geqslant \max _{1 \leqslant i \leqslant r-1}\left\{m_{i}\right\}$.

Theorem 16. Let $H$ be an $n$-admissible list with associated canonical list $\hat{H}=\left(h_{1}, \ldots, h_{r}\right)$ such that $r \geqslant 3, h_{r} \geqslant 2(r-1), h_{1}+\cdots+h_{r} \leqslant 2(n+r-2)$, and, if $r$ is even, $r \neq 4$ and $h_{1} \leqslant r$. Then $K_{n}^{*}$ is $H$-decomposable. 
Proof. We begin by defining a list $K=\left(k_{1}, k_{2}, \ldots, k_{r}\right)$ by permuting the elements of $\hat{H}$ with the purpose of ensuring that $K$ satisfies the inequalities (1) and (2) below, while retaining the property that $k_{r} \geqslant \max _{1 \leqslant i \leqslant r-1}\left\{k_{i}\right\}$.

(1) If $r$ is odd, then $k_{1}+k_{3}+\cdots+k_{r-2}+k_{r} \geqslant k_{2}+k_{4}+\cdots+k_{r-1}+(r+1)$.

(2) If $r$ is even, then $k_{2}+k_{4}+\cdots+k_{r-2}+k_{r} \geqslant k_{1}+k_{3}+\cdots+k_{r-1}+(r-2)$.

If $r$ is odd, take $k_{r}=h_{r}$, and define $k_{1}, \ldots, k_{r-1}$ by

$$
\left(k_{2}, k_{4}, \ldots, k_{r-1}\right)=\left(h_{1}, h_{2}, \ldots, h_{(r-1) / 2}\right)
$$

and

$$
\left(k_{1}, k_{3}, \ldots, k_{r-2}\right)=\left(h_{(r+1) / 2}, h_{(r+3) / 2}, \ldots, h_{r-1}\right) .
$$

This ensures that the elements of $\left(k_{1}, \ldots, k_{r}\right)$ with odd index are greater than or equal to those with even index, i.e. $k_{2 i-1} \geqslant k_{2 i}$ for $i \in\{1, \ldots,(r-1) / 2\}$. Since $k_{r} \geqslant 2(r-1)$, it is now easy to see that (1) is satisfied.

If $r$ is even, take $k_{r}=h_{r}, k_{r-1}=h_{4}, k_{r-2}=h_{3}, k_{r-3}=h_{2}$ and $k_{1}=h_{1}$, and define $k_{2}, \ldots, k_{r-4}$ so that

$$
\left(k_{3}, k_{5}, \ldots, k_{r-5}\right)=\left(h_{5}, h_{6} \ldots, h_{(r+2) / 2}\right)
$$

and

$$
\left(k_{2}, k_{4}, \ldots, k_{r-4}\right)=\left(h_{(r+4) / 2}, h_{(r+6) / 2}, \ldots, h_{r-1}\right) .
$$

Thus, $k_{r}=\max \left\{k_{1}, \ldots, k_{r}\right\}, k_{r-2} \geqslant k_{r-3}, k_{r-4} \geqslant k_{r-1}$, and $k_{2 i} \geqslant k_{2 i+1}$ for $i \in\{1, \ldots,(r-$ $6) / 2\}$. Since the elements of $H$ are written in nondecreasing order, using the assumptions that $k_{r}=h_{r} \geqslant 2(r-1)$ and $k_{1}=h_{1} \leqslant r$, it is now easy to check that (2) is satisfied.

We now define lists $\left(m_{1}, \ldots, m_{r}\right)$ and $\left(s_{0}, \ldots, s_{r}\right)$ of integers with $m_{r} \geqslant \max _{1 \leqslant i \leqslant r-1}\left\{m_{i}\right\}$ satisfying the conditions of Theorem 15, such that for each $i \in\{1, \ldots, r\}, k_{i}=m_{i}+s_{i-1}+$ $s_{i}$. The inequalities (1) and (2) will ensure that $m_{r-2} \geqslant 3$.

Note that by Lemma 10 and the assumptions that $k_{r}=h_{r} \geqslant 2(r-1)$ and $r \geqslant 3$, we have

$$
3(r-1) \leqslant k_{r}+2(r-2) \leqslant k_{1}+\cdots+k_{r-1} .
$$

Thus, we can find integers $m_{1}, \ldots, m_{r}$ such that $3 \leqslant m_{i} \leqslant k_{i}$ for each $i \in\{1, \ldots, r\}$ and $m_{1}+\cdots+m_{r-1}=k_{r}+2(r-2)$. Specifically, define

$$
t=\frac{k_{1}+k_{2}+\cdots+k_{r-1}-k_{r}-2(r-2)}{2},
$$

and note that $t$ is a non-negative integer by Lemma 10 .

We define a sequence of non-negative integers $s_{0}, s_{1}, s_{2}, \ldots, s_{r}$ by $s_{0}=s_{r-1}=s_{r}=0$, for $i \in\{1,2, \ldots, r-3\}$, set

$$
s_{i}=\min \left\{k_{i}-s_{i-1}-3, t-\left(s_{1}+\cdots+s_{i-1}\right)\right\},
$$

and define $s_{r-2}=t-\left(s_{1}+\cdots+s_{r-3}\right)$. It is evident from the definition that $s_{i-1}+s_{i} \leqslant k_{i}-3$ for each $i \in\{1, \ldots, r-3\}$ and $s_{1}+s_{2}+\cdots+s_{r}=t$. 
We now show by induction that $s_{i} \geqslant 0$ for each $0 \leqslant i \leqslant r$. First note that $s_{0}=$ $0 \geqslant 0$ and $s_{1}=\min \left\{k_{1}-3, t\right\} \geqslant 0$. Now suppose that $i>1$ and assume as the inductive hypothesis that $s_{j} \geqslant 0$ for each $0 \leqslant j<i$. Firstly, if $s_{i}=k_{i}-s_{i-1}-3$ then $s_{i-1}+s_{i-2} \leqslant k_{i-1}-3$, so $s_{i-1} \leqslant k_{i-1}-3$, which implies that $k_{i}-s_{i-1}-3 \geqslant 0$, since $k_{i} \geqslant k_{i-1}$. Alternatively if $s_{i}=t-\left(s_{1}+\cdots+s_{i-1}\right)$, then by the definition of $s_{i-1}$, $s_{i-1} \leqslant t-\left(s_{1}+\cdots+s_{i-2}\right)$ which implies $t-\left(s_{1}+\cdots+s_{i-1}\right) \geqslant 0$. Hence, noting that $s_{r-1}=s_{r}=0$, each $s_{i} \geqslant 0$.

For each $i \in\{1, \ldots, r\}$, set $m_{i}=k_{i}-s_{i-1}-s_{i}$. By choice of $s_{r-2}$, it is easy to see that

$$
s=\sum_{i=1}^{r-1} s_{i}=t
$$

and hence

$$
\sum_{i=1}^{r-1} m_{i}=\sum_{i=1}^{r-1} k_{i}-2 \sum_{i=1}^{r-1} s_{i}=\sum_{i=1}^{r-1} k_{i}-2 t=k_{r}+2(r-2)=m_{r}+2(r-2) .
$$

It is now easy to check that $\left(m_{1}, \ldots, m_{r}\right)$ satisfy conditions 1 and 2 of Lemma 10. Also, since $m_{i} \leqslant k_{i}$ for each $i \in\{1, \ldots, r\}$, we have that each $m_{i} \leqslant k_{i} \leqslant n$, and $m_{1}+\cdots+m_{r} \leqslant$ $k_{1}+\cdots+k_{r} \leqslant n(n-1)$. Thus, to show that $\left(m_{1}, \ldots, m_{r}\right)$ can be viewed as the (suitably ordered) canonical list of an $n$-admissible list $M$, we need only to show that $m_{i} \geqslant 3$ for each $i \in\{1, \ldots, r\}$. This is clear if $1 \leqslant i \leqslant r-3$ since $s_{i} \leqslant k_{i}-s_{i-1}-3$, and if $i=r$ since $m_{r}=k_{r}$.

For $i=r-2$, we have

$$
m_{r-2}=k_{r-2}-s_{r-3}-s_{r-2} .
$$

If $s_{r-2}=0$, then $m_{r-2}=k_{r-2}-s_{r-3} \geqslant k_{r-3}-s_{r-3} \geqslant m_{r-3} \geqslant 3$. Otherwise,

$$
\begin{aligned}
m_{r-2} & =k_{r-2}-s_{r-3}-s_{r-2} \\
& =k_{r-2}-s_{r-3}-\left(t-\left(s_{1}+s_{2}+\cdots+s_{r-3}\right)\right) \\
& =k_{r-2}-t+s_{1}+s_{2}+\cdots+s_{r-4} .
\end{aligned}
$$

To bound the value of $m_{r-2}$, we rewrite this quantity in terms of $k_{1}, \ldots, k_{r}$. Note that for each $j \in\{1, \ldots, r-3\}$, if $s_{j}=t-\left(s_{1}+\cdots+s_{j-1}\right)$, then $s_{j+1}=0$. Hence the condition $s_{r-2} \neq 0$ implies that $s_{j}=k_{j}-s_{j-1}-3$ for each $j \in\{1, \ldots, r-3\}$. Thus

$$
\begin{aligned}
& s_{1}+\cdots+s_{r-4}=\sum_{i=1}^{r-4}\left(k_{i}-s_{i-1}-3\right) \\
& =\left(k_{1}-3\right)+\left[\left(k_{2}-3\right)-\left(k_{1}-3\right)\right]+\left[\left(k_{3}-3\right)-\left(k_{2}-3\right)+\left(k_{1}-3\right)\right]+\cdots \\
& +\left[\left(k_{r-4}-3\right)+\cdots+(-1)^{r-5}\left(k_{1}-3\right)\right] \\
& = \begin{cases}k_{1}+k_{3}+\cdots+k_{r-4}-3(r-3) / 2 & \text { if } r \text { is odd } \\
k_{2}+k_{4}+\cdots+k_{r-4}-3(r-4) / 2 & \text { if } r \text { is even. }\end{cases}
\end{aligned}
$$


If $r$ is odd, it follows that

$$
\begin{aligned}
m_{r-2} & =k_{r-2}-t+s_{1}+\cdots+s_{r-4} \\
& =k_{1}+k_{3}+\cdots+k_{r-4}+k_{r-2}-\frac{3(r-3)}{2}-\frac{k_{1}+k_{2}+\cdots+k_{r-1}-k_{r}-2(r-2)}{2} \\
& =\frac{k_{1}+k_{3}+\cdots+k_{r-2}+k_{r}}{2}-\frac{k_{2}+k_{4}+\cdots+k_{r-1}}{2}-\frac{r-5}{2} \\
& \geqslant 3,
\end{aligned}
$$

since $k_{1}+k_{3}+\cdots+k_{r-2}+k_{r} \geqslant k_{2}+k_{4}+\cdots+k_{r-1}+r+1$.

If $r$ is even, then

$$
\begin{aligned}
m_{r-2} & =k_{r-2}-t+s_{1}+\cdots+s_{r-4} \\
& =k_{2}+k_{4}+\cdots+k_{r-4}+k_{r-2}-\frac{3(r-4)}{2}-\frac{k_{1}+k_{2}+\cdots+k_{r-1}-k_{r}-2(r-2)}{2} \\
& =\frac{k_{2}+k_{4}+\cdots+k_{r-2}+k_{r}}{2}-\frac{k_{1}+k_{3}+\cdots+k_{r-1}}{2}-\frac{r-8}{2} \\
& \geqslant 3
\end{aligned}
$$

since $k_{2}+k_{4}+\cdots+k_{r-2}+k_{r} \geqslant k_{1}+k_{3}+\cdots+k_{r-1}+r-2$.

Finally, we show that $m_{r-1} \geqslant 3$. Since $s_{r-1}=0$ and by construction $k_{r-1} \geqslant k_{r-2}$, we have that

$$
m_{r-1}=k_{r-1}-s_{r-2} \geqslant k_{r-2}-s_{r-2}-s_{r-3}=m_{r-2} \geqslant 3 .
$$

We now show that the list satisfies Condition 2 of Theorem 15, i.e. that $m_{r}+s \leqslant n$. Since

$$
s=\sum_{i=1}^{r-1} s_{i}=\sum_{i=1}^{r-1}\left(k_{i}-m_{i}-s_{i-1}\right)=\left(\sum_{i=1}^{r-1} k_{i}\right)-\left(k_{r}+2(r-2)\right)-s,
$$

we have

$$
2 s=\left(\sum_{i=1}^{r} k_{i}\right)-2 k_{r}-2(r-2),
$$

giving

$$
s=\frac{1}{2}\left(\sum_{i=1}^{r} k_{i}\right)-k_{r}-r+2 \leqslant(n+r-2)-k_{r}-r+2=n-k_{r},
$$

so that $m_{r}+s=k_{r}+s \leqslant n$.

The result now follows by applying Theorem 15 , taking $\hat{M}^{\prime}=K$.

We note that the conclusion of Theorem 16 holds whenever $r \geqslant 3, h_{r} \geqslant 2(r-1)$, $h_{1}+\cdots+h_{r} \leqslant 2(n+r-2)$ and there is a reordering of the $h_{i}, 1 \leqslant i<r$, so that inequalities (1) and (2) are satisfied. In particular, when $r$ is even, the conditions $r \neq 4$ and $h_{1} \leqslant r$ may be dropped if (2) holds in the reordering of the $h_{i}$.

Recall that a directed cycle decomposition of $K_{n}^{*}$ can be considered as a decomposition of $G^{*}$ into directed cycles of lengths greater than 2 for some graph $G$ of order $n$. Theorem 16 applies when the underlying (undirected) graph $G$ formed by cycles not of length 
two is sparse. In particular, it solves all cases where $G$ has one vertex of degree $r \geqslant 5$ $(r \geqslant 3$ if $r$ is odd) and all the rest of degree at most three, and which is a subdivision of a Hamiltonian graph. It thus gives a solution in all cases where $G$ can be decomposed into subgraphs which either have this form or are 2-regular.

\section{Admissible lists with three cycles of length greater than 2}

Recall that Theorem 13 states that $K_{n}^{*}$ is $M$-decomposable whenever $M$ is an admissible list containing at most two cycles of length greater than 2 . In this section we give necessary and sufficient conditions for the existence of an $M$-decomposition of $K_{n}^{*}$ in the case that the associated canonical list $\hat{M}$ has size 3 . We begin by noting the following special case of Lemma 10.

Lemma 17. If $M$ is an $n$-admissible list with associated canonical list $\hat{M}=(\alpha, \beta, \gamma)$, then $\alpha+\beta \equiv \gamma(\bmod 2)$ and $\alpha+\beta>\gamma$.

For the case that $\alpha+\beta+\gamma \leqslant 2(n+1)$, sufficiency follows directly from Theorem 16 . Specifically, we have the following lemma.

Lemma 18. Let $M$ be an $n$-admissible list with associated canonical list $\hat{M}=(\alpha, \beta, \gamma)$. If $\alpha+\beta+\gamma \leqslant 2(n+1)$, then $K_{n}^{*}$ is $M$-decomposable.

Proof. Note that the conditions of Theorem 16 when $r=3$ are that $\alpha+\beta+\gamma \leqslant 2(n+1)$ (as in the assumption) and $\gamma \geqslant 4$. However, if $\gamma=3$, then $\alpha=\beta=\gamma=3$, so that $\alpha+\beta \not \equiv \gamma(\bmod 2)$, in contradiction to Lemma 17 .

If $\hat{M}=(\alpha, \beta, \gamma)$ and $\alpha+\beta+\gamma>2(n+1)$, the existence of an $M$-decomposition of $K_{n}^{*}$ depends on the value of $\gamma$ as well as the congruence classes of $\alpha+\beta$ and $\gamma$ modulo 4 .

Lemma 19. Let $M$ be an $n$-admissible list with associated canonical list $\hat{M}=(\alpha, \beta, \gamma)$. If $\alpha+\beta+\gamma>2(n+1)$ and $\alpha+\beta \not \equiv \gamma(\bmod 4)$, then $K_{n}^{*}$ is $M$-decomposable.

Proof. We construct directed cycles of lengths $\alpha, \beta$ and $\gamma$, leaving no decoupled arcs, so that the remaining arcs can be used to form directed 2-cycles by Lemma 8. By Lemma 12, it is sufficient to consider the case $\gamma=n$.

Let $\vec{C}_{n}=(0, n-1, n-2, \ldots, 1)$. To construct $\vec{C}_{\alpha}$ and $\vec{C}_{\beta}$, we must use the decoupled arcs along the directed cycle $\vec{C}=(0,1, \ldots, n-1)$, together with $t=(\alpha+\beta-n) / 2$ further pairs of arcs. Note that $t$ is odd and $t>1$.

We first form the cycle of length $\beta$. Let $\ell=n-\beta$. If $t=3$, let

$$
\vec{C}_{\beta}=(0, \ell+3, \ell+4, \ell+1, \ell+2, \ell+5, \ell+6, \ell+7, \ldots, n-1) .
$$

Otherwise, we build $\vec{C}_{\beta}$ by concatenating directed paths. Let

$$
P=[0, \ell+3, \ell+4, \ell+1, \ell+2] .
$$


For each $i=0,1, \ldots,(t-5) / 2$, let

$$
P_{i}=[\ell+4 i+2, \ell+4 i+7, \ell+4 i+8, \ell+4 i+5, \ell+4 i+6] .
$$

Note that:

- The initial vertex of $P_{0}$ is $\ell+2$, the terminal vertex of $P$.

- For each $i=1,2, \ldots,(t-5) / 2$, the terminal vertex of $P_{i-1}$ and the initial vertex of $P_{i}$ coincide. Moreover, the paths $P, P_{0}, P_{1}, \ldots, P_{(t-5) / 2}$ are pairwise internally vertex-disjoint, so that the concatenation $P P_{0} P_{1} \cdots P_{(t-5) / 2}$ is a path.

- Each $P_{i}$ and $P$ uses two arcs of $\vec{C}$ and two other arcs. In total, these paths contain $(t-1) \operatorname{arcs}$ of $\vec{C}$ and decouple $(t-1)$ further arcs.

Next let $Q=[\ell+2 t-4, \ell+2 t-1]$. Note that the initial vertex of $Q$ is the terminal vertex of $P_{(t-5) / 2}$, and the terminal vertex of $Q$ is $\ell+2 t-1=\alpha-1 \leqslant n-1$, which has not occurred in any of the paths so far. Finally, let $Q^{\prime}=[\ell+2 t-1, \ell+2 t, \ldots, \ell+2 t-1+(n-2 t-\ell+1)]$. Since the terminal vertex of $Q^{\prime}$ is $\ell+2 t-1+n-2 t-\ell+1=n=0$, it is now easy to see that the concatenation

$$
P P_{0} P_{1} \cdots P_{(t-5) / 2} Q Q^{\prime}
$$

forms a directed cycle of length

$$
4+4(t-3) / 2+1+(n-2 t-\ell+1)=n-\ell=\beta .
$$
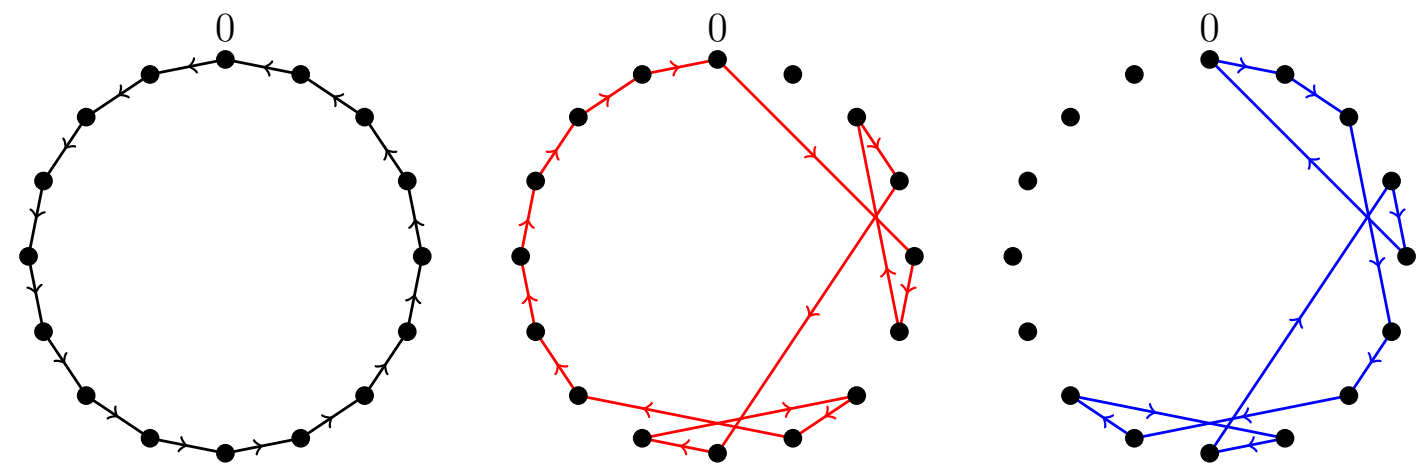

Figure 3: An illustration of the construction of Lemma 19. Here $\alpha=11, \beta=15$, $\gamma=16=n$.

It is not difficult to show that the remaining decoupled arcs form a cycle of length $\alpha$; see Figure 3.

Lemma 20. Let $M$ be an n-admissible list with associated canonical list $\hat{M}=(\alpha, \beta, \gamma)$. If $\alpha+\beta+\gamma>2(n+1), \alpha+\beta \equiv \gamma(\bmod 4)$ and $\gamma<n$, then $K_{n}^{*}$ is $M$-decomposable. 
Proof. First, note that $\alpha \geqslant 4$, as otherwise $2(n+1)<\alpha+\beta+\gamma \leqslant 3+\beta+(n-1)=\beta+n+2$, implying $\beta>n$. Let $M^{\prime}$ be the $(n-1)$-admissible list with associated canonical list $\hat{M}^{\prime}=(\alpha-1, \beta-1, \gamma)$.

Since $\alpha+\beta+\gamma>2(n+1)$, we have that $(\alpha-1)+(\beta-1)+\gamma>2 n$, and so by Lemma $19, K_{n-1}^{*}$ is $M^{\prime}$-decomposable. Consider the cycles $\vec{C}_{\alpha-1}, \vec{C}_{\beta-1}$ and $\vec{C}_{\gamma}$ in the decomposition constructed by Lemma 19. It is easy to see that there exist arcs $x y$ and $y x$ with $x y \in \vec{C}_{\alpha-1}$ and $y x \in \vec{C}_{\beta-1}$. Add a new vertex $z$, and replace arcs $x y$ and $y x$ with directed paths $x z y$ and $y z x$. We thus obtain three cycles of lengths $\alpha, \beta$ and $\gamma$ in $K_{n}^{*}$ which between them leave no arc decoupled. The result follows by Lemma 12 .

Note that the conditions of Lemma 20 require that the largest cycle be nonHamiltonian. Indeed, if $\gamma=n$ but all other conditions remain the same as those of Lemma 20, no $M$-decomposition exists. To prove this result, we will exploit a connection between an $M$-decomposition with $\hat{M}=(\alpha, \beta, n)$ and perfect 1 -factorizations, which we now define.

Definition 21. A 1-factorization $\mathcal{F}$ of a graph $G$ is a perfect 1 -factorization if, for any two 1 -factors $F_{1}$ and $F_{2}$ in $\mathcal{F}, F_{1} \cup F_{2}$ is a Hamiltonian cycle.

We will need the following result on perfect 1-factorizations of cubic bipartite graphs, due to Kotzig and Labelle [7].

Lemma $22([7])$. Let $G$ be a cubic bipartite graph with bipartition $(X, Y)$, where $|X|=$ $|Y|=t$. If $G$ admits a perfect 1-factorization, then $t$ is odd.

Lemma 23. Let $M$ be an $n$-admissible list with associated canonical list $\hat{M}=(\alpha, \beta, n)$. If $\alpha+\beta+n>2(n+1)$ (i.e. $\alpha+\beta>n+2)$ and $\alpha+\beta \equiv n(\bmod 4)$, then $K_{n}^{*}$ is not $M$-decomposable.

Proof. Suppose that such a decomposition exists, and let $\vec{C}=(n-1, n-2, \ldots, 1,0)$ be the directed $n$-cycle in the decomposition. Colour the edges of the directed $\alpha$-cycle green and those of the directed $\beta$-cycle red, and without loss of generality, suppose that the arc 01 is green. Note that each arc of $\overleftarrow{C}=(0,1, \ldots, n-1)$, the reversal of $\vec{C}$, must be coloured green or red; in fact, $\overleftarrow{C}$ is partitioned into an equal number of green and red directed paths, say $G_{0}, G_{1}, \ldots, G_{t-1}$ and $R_{0}, R_{1}, \ldots, R_{t-1}$, where the green path $G_{i}$ has initial and terminal vertices $x_{i}$ and $y_{i}$, and the red path $R_{i}$ has initial and terminal vertices $y_{i}$ and $x_{i+1}$ (where $x_{t+1}=x_{0}$ ), and $t=\frac{1}{2}(\alpha+\beta-n)$ is the number of edges of $K_{n}^{*}$ which are in the cycles of length $\alpha$ and $\beta$ but not in $\overleftarrow{C}$

We form a bipartite graph $B$ with partite sets $\left\{x_{0}, x_{1}, \ldots, x_{t-1}\right\}$ and $\left\{y_{0}, y_{1}, \ldots, y_{t-1}\right\}$ as follows. For $i=0,1, \ldots, t-1$, we join $x_{i}$ to $y_{i}$, forming a 1 -factor $F_{1}$ of $B$, and $y_{i}$ to $x_{i+1}$ (computing subscripts modulo $t$ ), forming a second 1 -factor $F_{2}$ of $B$. Also, join $y_{i}$ to $x_{j}$, where $y_{i} x_{j}$ is an arc of the green cycle; these edges form a third 1-factor $F_{3}$ of $B$. Note that $j \neq i+1$, as otherwise the $\alpha$-cycle in the decomposition must be $\left(y_{i}, y_{i}+1, \ldots, y_{i+1}\right)$, implying $t=1$ and hence $\alpha+\beta-n=2 \equiv 2(\bmod 4)$. An example of the $\alpha$ - and $\beta$-cycles together with the associated cubic bipartite graph can be found in Figure 4. 

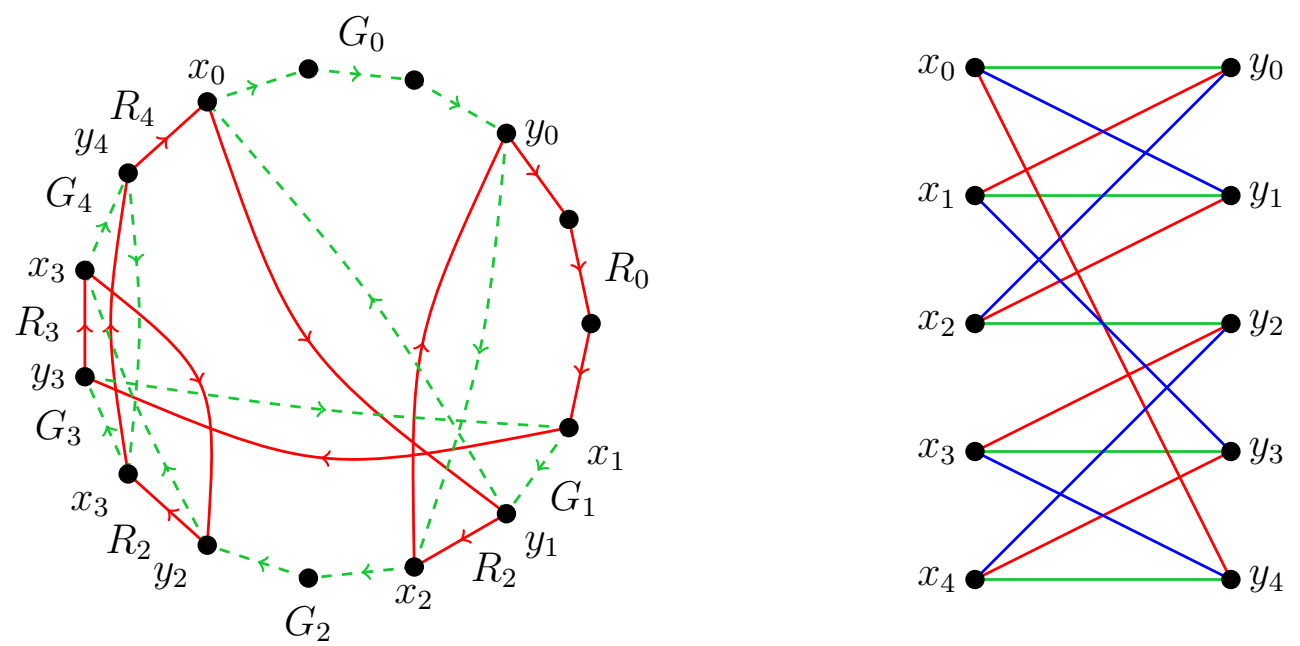

Figure 4: Directed $\alpha$ - and $\beta$-cycles together with the associated cubic bipartite graph

It is clear that $B$ is a cubic bipartite graph. We show that $\left\{F_{1}, F_{2}, F_{3}\right\}$ is a perfect 1-factorization of $B$. First, notice that $F_{1} \cup F_{2}$ induces the Hamiltonian cycle $\left(x_{0}, y_{0}, x_{1}, y_{1}, \ldots, x_{t-1}, y_{t-1}\right)$. Next, consider the green $\alpha$-cycle in the decomposition. Replacing the arcs of each $G_{i}$ with a single arc $x_{i} y_{i}$ and disregarding direction, we obtain a cycle whose edges correspond to those of $F_{1}$ and $F_{3}$, and so $F_{1} \cup F_{3}$ induces a Hamiltonian cycle in $B$. In a similar way, looking at the red $\beta$-cycle shows that $F_{2} \cup F_{3}$ induces a Hamiltonian cycle in $B$.

By Lemma 22, the existence of a perfect 1-factorization of $B$ implies that $t$ is odd, contradicting the fact that $\alpha+\beta-n \equiv 0(\bmod 4)$.

Summarizing the results of this section, we have the following complete result for canonical lists of size at most three.

Theorem 24. Let $M=(2,2, \ldots, 2, \alpha, \beta, \gamma)$, where $2<\alpha \leqslant \beta \leqslant \gamma \leqslant n$. The complete symmetric digraph $K_{n}^{*}$ is $M$-decomposable if and only if $M$ is $n$-admissible and it is not the case that $\alpha+\beta>n+2, \gamma=n$ and $\alpha+\beta \equiv n(\bmod 4)$.

One immediate consequence is the following result regarding cycle decomposition of certain 3-regular digraphs.

Corollary 25. Let $\alpha, \beta, \gamma \geqslant 3$ be integers with $\alpha+\beta>\gamma+2$ and $\alpha+\beta \equiv \gamma(\bmod 4)$. If $G$ is a 3-regular graph with $\gamma$ vertices and $(\alpha+\beta+\gamma) / 2$ edges, then $G^{*}$ is not $(\alpha, \beta, \gamma)$ decomposable.

\section{Conclusion}

In this paper, we have made progress on the problem of decomposing a complete symmetric digraph into cycles of given lengths. Theorem 16 shows that if the greatest cycle length 
and the number of 2-cycles in an $n$-admissible list $M$ are both large enough, then $K_{n}^{*}$ admits an $M$-decomposition.

In the case that there are at most three cycles of length greater than 2 , we have given a complete solution (Theorem 24). Notably, there is a family of $n$-admissible lists $M$, namely those with $\hat{M}=(\alpha, \beta, n)$ where $\alpha+\beta>n+2$ and $\alpha+\beta \equiv n(\bmod 4)$, for which $2 K_{n}$ is decomposable but $K_{n}^{*}$ is not. This result implies that no cubic graph $G$ of order $n$ exists such that $G^{*}$ is decomposable into three cycles of lengths $\alpha, \beta$ and $n$, where $\alpha+\beta>n+2$ and $\alpha+\beta \equiv n(\bmod 4)$. In particular, $G^{*}$ has no Hamiltonian cycle decomposition if $n \equiv 0(\bmod 4)$.

The method used in this paper to show non-existence by constructing an auxiliary bipartite graph with a perfect 1-factorization does not apply in general to canonical lists of size greater than three. It remains an interesting open question to determine if there are other infinite families of $n$-admissible lists $M$ for which $K_{n}^{*}$ is not $M$-decomposable. We have checked all $n$-admissible lists with $n \leqslant 13$ and have verified that the corresponding decompostions exist, except for those given by Lemma 23 and the decompositions corresponding to the canonical lists:

$$
\begin{array}{ll}
n=4: & (4,4,4) \\
n=5: & (4,4,5,5),(3,4,4,4,5),(3,3,3,3,3,5) ; \\
n=6: & (3,3,3,3,3,3,3,3,3,3),(3,3,6,6,6,6),(4,6,6,6),(6,6,6,6,6),
\end{array}
$$

which do not exist. We conjecture that these are the only exceptions.

\section{References}

[1] B. Alspach. Research Problem 3. Discrete Math. 36 (1981), 333.

[2] B. Alspach and H. Gavlas. Cycle decompositions of $K_{n}$ and $K_{n}-I$. J. Combin. Theory Ser. B 81 (2001), 77-99.

[3] B. Alspach, H. Gavlas, M. Šajna and H. Verrall. Cycle decompositions IV: complete directed graphs and fixed length directed cycles. J. Combin. Theory Ser. A 103 (2003), 165-208.

[4] D. Bryant, D. Horsley, B. Maenhaut and B.R. Smith. Decompositions of complete multigraphs into cycles of varying lengths. J. Combin. Theory Ser. B 129 (2018), 79-106.

[5] D. Bryant, D. Horsley and W. Pettersson. Cycle decompositions V: Complete graphs into cycles of arbitrary lengths. Proc. London Math. Soc. 108 (2014), 1153-1192.

[6] T.P. Kirkman. On a problem in combinations. Cambridge and Dublin Math J. 2 (1847), 191-204.

[7] A. Kotzig and J. Labelle. Strongly Hamiltonian graphs. Utilitas Math. 14 (1978), 99-116.

[8] E. Lucas. Récréations Mathématiques, Vol. II. Gauthier-Villars, Paris, 1892.

[9] M. Šajna. Cycle decompositions III: Complete graphs and fixed length cycles. J. Combin. Des. 10 (2002), 27-78. 